\title{
The automatic interpretation of structural plane parameters in borehole camera images from drilling engineering
}

\author{
Chuanying Wang ${ }^{1{ }^{\dagger}}$ Xianjian Zou ${ }^{1) 2}$ Zengqiang Han ${ }^{1)}$ Jinchao Wang ${ }^{1)}$ Yiteng Wang ${ }^{1)}$ \\ 1) State Key Laboratory of Geomechanics and Geotechnical Engineering, Institute of Rock and \\ Soil Mechanics, Chinese Academy of Sciences, Wuhan 430071, China \\ 2) School of Electronic Information, Wuhan University, 430072, Wuhan, China
}

\begin{abstract}
Digital panoramic borehole camera technology has been widely employed in actual projects, and a large number of high-accuracy borehole camera images have been obtained. Borehole camera images accurately record the geological information, especially the feature parameters of structure plane. However, since the acquisition of these features is usually done by hand, the workload is large and the results can be affected by human error. To solve this problem, this paper presents an automatic interpretation method of structure plane parameters in borehole camera image. In this method, image pixel gray, gradient values and projection method are employed to distinguish the occurrence region of structural planes. Then, standard sine function matching method is employed to search the structure plane in the region. Lastly, the optimal sine curve is screened out and adopted as the feature curve of structure plane. Related parameters of sine curve are analyzed and converted into the parameters of structure plane, such as the central position, orientation, dip angle and fracture width of structural planes. This method can automatically identify structure planes in the image continuously and quickly, and obtain the corresponding structural parameters. The method is stable and reliable, and greatly improves the working efficiency. It can perform the automatic interpretation of structure planes and extraction of geometric parameters, and provide an effective and consistent solution for drilling information acquisition and borehole camera image signal processing.
\end{abstract}

Keywords: automatic interpretation; borehole camera image; structure plane; regional division; sine curve

Corresponding author: Chuanying Wang

E-mail: chywang@whrsm.ac.cn, Tel: +8613517105001

Corresponding E-mail: zouxianjian@whu.edu.cn 


\section{Nomenclature}

\begin{tabular}{|c|c|}
\hline Pos & Position of structure plane \\
\hline$\alpha$ & Orientation of structure plane \\
\hline$\beta$ & Dip angle of structure plane \\
\hline$d$ & Fracture width of structure plane \\
\hline$j_{0}$ & Initial coordinate position of sine curve \\
\hline$\theta$ & Phase angle of sine curve \\
\hline$A$ & Amplitude of sine curve \\
\hline$D$ & Borehole diameter \\
\hline $\mathrm{W}$ & Width of each sub-block \\
\hline$M$ & Number of the whole composited signal \\
\hline $\operatorname{Min} V(j)$ & Minimum gray value function \\
\hline $\operatorname{Max} V(j)$ & Maximum gray value function \\
\hline $\operatorname{Max} G(j)$ & Maximum gradient value function \\
\hline $\operatorname{ComV}(j)$ & Composited signal function of characteristic values \\
\hline $\operatorname{Com} T(j)$ & Sub-block signal function \\
\hline $\operatorname{Max} N(j)$ & Total number function of matched points \\
\hline$y(i)$ & Template sine curve function \\
\hline$T_{\text {gary }}$ & Judging threshold of pixel value whether belongs to structure plane \\
\hline$\overline{\operatorname{ComT}_{\text {global }}}$ & The global threshold of the composited signal \\
\hline$\overline{\operatorname{Com} T_{\text {local }}}$ & The local threshold of the composited signal \\
\hline
\end{tabular}

\section{Introduction}

Digital panoramic borehole camera system is capable of simultaneously observing 360 degrees of drilling hole, and has been widely employed in geotechnical, civil, mining, hydropower engineering and geological disaster prevention projects (Bae et al., 2011; Cunningham et al., 2004; Genter et al., 1997; Williams and Johnson, 2004; Zohreh et al., 2014). This system can obtain a large number of high-precision borehole camera images, which accurately record the morphological characteristics and geometrical parameters of discontinuities or structural plane in borehole wall (Han et al., 2013; Wang et al., 2002; Wang et al., 2010). For drilling engineering, the post processing of the borehole camera image for obtaining structural plane 
parameters are often short of a fast and effective method, leading to large consumption of manpower and material resources (Lofi et al., 2012; Schepers et al., 2001). At present, the parameter extraction of structural planes' features in borehole camera images is basically reliant on the manual operation and subjective interpretation (Assous et al., 2014; Hurich and Deemer, 2013). Therefore, the exactly automatic interpretation of structural planes' morphological characteristics parameters in borehole camera images is of great practical significance, which is also conducive to the further development of borehole imaging technology.

In this paper, a new automatic interpretation method of structural planes in borehole camera image is proposed. It can perform intelligent extraction of structural parameters, such as the central position, the orientation and the dip angle of structural planes. In this method, the image pixel gray and gradient characteristics from the optical imaging of borehole wall are used to form new signals for the automatic interpretation of structural planes, such as the maximum, minimum gray value and the maximum gradient value of each line in the image. In borehole camera image, since the structure plane is similar as a shape of sine curve or similar to the geometry of sine curve, this method employs the template sinusoidal function to carry out iterative matching operation. We choose the best fitting sine curve as the geometric curve of structure plane and its corresponding geometric parameters are obtained from the best fitting sine curve. So, the automatic interpretation of structure planes in borehole camera image is performed by this method.

\section{Digital panoramic borehole camera images}

Borehole camera images are the indirect reflection of drilling hole-wall. The structure and composition of drilling hole-wall determine the imaging feature of the borehole camera image. Therefore, the borehole camera image is consisted of two parts: one is the imaging of rock, and the other is the imaging of structure planes or discontinuities. Due to the inherently different properties of rocks, the color and depth of different rocks and discontinuities in borehole camera image are different. When the rock (lamprophyre) or minerals (biotite, amphibole and pyroxene, etc.) is dark or the reflection is poor, borehole camera image is dim; when light colored rocks or minerals (feldspar, quartz and muscovite) is strong, the camera image is bright (Djurhuus and Aadn O Y, 2003; Prensky, 1999). Furthermore, the structure plane of 
drilling hole-wall is often shown as a black ribbon of hole or curve region, which is relatively prominent.

When the probe of borehole camera is put into the drilling or borehole, the micro CCD camera receives the reflection ray of the borehole wall, which is varied due to different reflection of different rock or filling material, hence the structure of the camera image has different lights and shades. If the structure plane is cemented by quartz feldspar, the hole-wall is complete, and the reflection of the incident light is good, then the camera image is close to the actual condition of the hole-wall. If the structure is filled with mud and sand, it will cause poor reflection and a dark camera image; if the structure is open and the incident light is not reflected back to the micro CCD camera, the structure plane of the borehole camera image is shown as black, as shown in Fig. 1, where Fig. 1a is a relatively independent simple rule structure plane, and Fig. $1 \mathrm{~b}$ is a complex irregular structure plane. In this paper, the borehole camera image refers to the expanded view of the cylindrical borehole wall camera images, which is obtained by our Digital Panoramic Borehole Camera System (DPBCS) from the Institute of Rock and Soil Mechanics, Chinese Academy of Sciences (Wang et al., 2002). Structure planes refer to the sine curve band in the expanded view of borehole camera image.

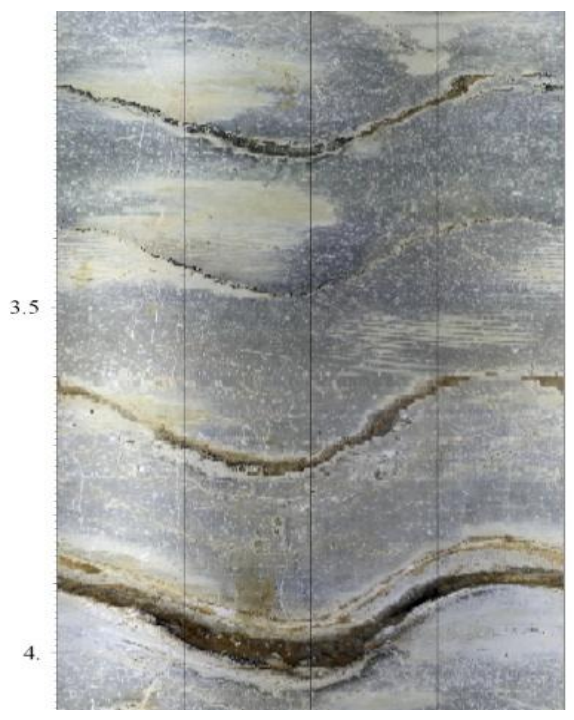

(a)

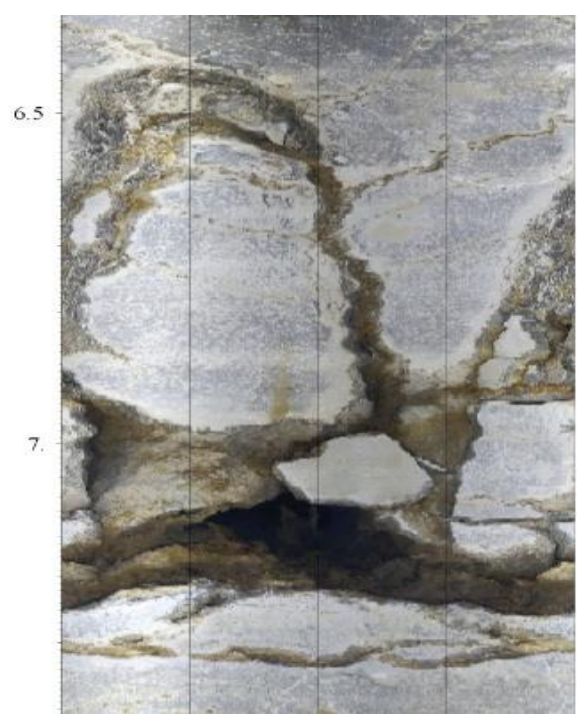

(b)

Fig. 1. Borehole camera images acquired by our Digital Panoramic Borehole Camera System (DPBCS), (a) Relatively independent, simple and regular structural planes, (b) Relatively crossed, complex and irregular structural planes.

\section{The automatic interpretation of structural planes}


Different structure planes show some differences due to different morphological and physical characteristics of rocks. In borehole camera images, color depth, shape variation and strip width are the main features of imaging structure planes. Because the structure plane is a 3D ellipse in actual drilling hole-wall, the standard structure plane is a standard sine curve band and the non-standard structure plane is also similar to a sine curve band in the expanding borehole image (Glossop et al., 1999; Thapa et al., 1997). Thus, the structure plane shapes in borehole image can be represented by a template sine function. In addition, structure planes can be described by using four parameters: depth or position Pos (i.e., the scanning line position), orientation $\alpha$ (i.e., the angle between nadir clockwise and the north direction), dip angle $\beta$ (i.e., the angle between of structure plane and the horizontal plane), and fracture width $d$ of structure plane. These parameters are corresponding to the coordinate position $j_{0}$, the phase angle $\theta$ and the amplitude $A$ of the sine curve in the borehole camera image.

According to the morphology of structure planes in borehole camera image, the manifestation of structure planes is similar to a sine curve band. Therefore, this paper uses template sine function to iterate through the points on the structural planes and surrounding area. Whether the iterated sine curve is consistent with the structure plane or not is determined by counting the number of the points which are satisfying the conditions. In addition, searching for a new characteristic value under the idea of clustering is employed to describe the structure plane, and the division of structure plane and the related signal processing and judgment. Then, combined with the idea of template sine function iterative matching, the automatic interpretation of geometric parameters can be achieved. The main steps of the method are as shown in Fig. 2.

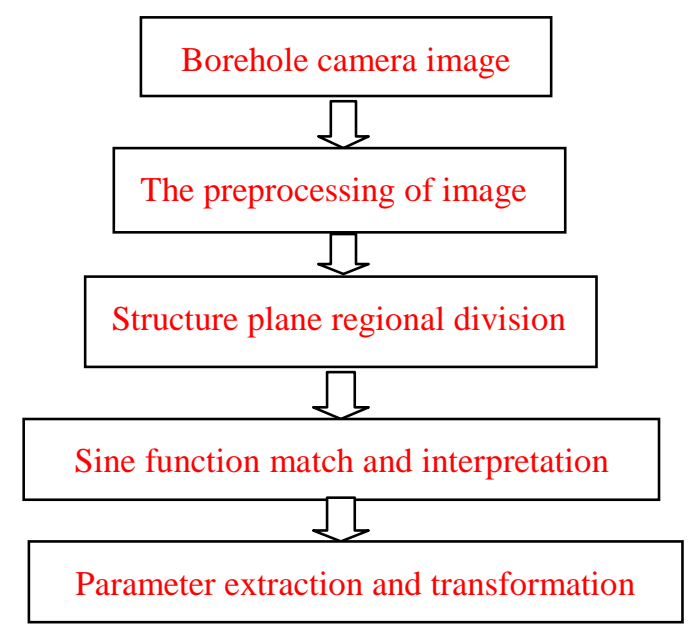

Fig. 2. The steps of automatic interpretation of structure plane parameters in borehole camera image. 


\subsection{The preprocessing of borehole camera image}

The sine curve band of the structure plane in borehole camera image is relatively obvious and bright, which is denoted as the target region. In the non-target region, because of the irregularity of borehole wall and the difference of rock properties, there are inevitably some separate light spots or feature regions in the image. These light spots and feature regions have great influence on the location and interpretation of the target region. However, the gray values of structure plane curves in the target region are often shown as the darkest pixels or the brightest pixels, and their distribution is relatively concentrated and continuously in the horizontal direction. If the projection is carried out in the vertical direction, these points of isolated structures will converge to a small region, and are relatively independent. Because most of the structure planes are not overlapped and they have relatively large intervals, the small region formed by projection can be directly classified as a whole, which is easily separated from the imaging noise of borehole wall.

In order to highlight the characteristics of the structure and avoid being covered by other independent points, this paper firstly describes the features of the structure by the minimum and maximum values of gray value of each line. The upper left corner in the camera image is denoted as the starting point of image and the pixel coordinates increase from top to bottom and from left to right. Borehole camera image is denoted as $f(x, y)$, and $f(i, j)$ is the gray value of the pixels at coordinate $(i, j)$. In the borehole camera image, the minimum gray value of each row is selected as the minimum eigenvalue of the row shown as Eq. (1), where $\operatorname{Min} V(j)$ is the minimum gray value of the $\mathrm{j}^{\text {th }}$ line. The maximum gray value of each row is selected as the maximum eigenvalue of the row shown as Eq. (2), where $\operatorname{Max} V(j)$ is the maximum gray value of the $\mathrm{j}^{\text {th }}$ line. The $N$ is a constant value is the width of borehole camera image. In this paper, $N$ is a constant 1024 .

$$
\begin{gathered}
\operatorname{Min} V(j)=\min [f(0, j), f(1, j), f(2, j), \cdots, f(i, j), \cdots, f(N-1, j)] \quad(i \in \mathbf{N}) . \\
\operatorname{Max} V(j)=\max [f(0, j), f(1, j), f(2, j), \cdots, f(i, j), \cdots, f(N-1, j)] \quad(i \in \mathbf{N}) .
\end{gathered}
$$

To further describe the gray level of structure plane and surrounding region, the gradient value is employed. We take the absolute gradient sum of neighborhoods $3 \times 3$ at coordinate $(i, j)$ as the gradient value shown in Eq. (3), where $G(i, j)$ is the gradient value of pixel point. So, the maximum gradient value of each row can be expressed as 
Eq. (4), where $\operatorname{Max} G(j)$ is the maximum gradient value of the $\mathrm{j}^{\text {th }}$ line.

$$
\begin{gathered}
G(i, j)=\sum_{k=-1}^{1}[f(i+k, j+1)-f(i+k, j-1)]+\sum_{k=-1}^{1}[f(i+1, j+k)-f(i-1, j+k)] . \\
M a x G(j)=\max [G(0, j), G(1, j), G(2, j), \cdots, G(i, j), \cdots, G(N-1, j)] \quad(i \in \mathbf{N}) .
\end{gathered}
$$

The minimum gray value and maximum gray value of each row represent the two pole regions in the borehole camera image. The sine curve bands of structure planes are made up of these poles (black spots and bright spots). These poles are generally distributed in or near the sine curve band of the structure plane, and their projection in the vertical direction is relatively concentrated. In order to avoid these poles from the non-target region, the difference of $\operatorname{Max} V(j)$ and $\operatorname{Min} V(j)$ is employed as the reference value of the row, and the maximum gradient value $\operatorname{Max} G(j)$ of the row or its multiple as the characteristic value of the composited signal $\operatorname{Com} S(j)$. The expression $\operatorname{ComS}(j)$ is shown in Eq. (5):

$$
\operatorname{ComS}(j)=\operatorname{Max} V(j)-\operatorname{Min} V(j)+\lambda \cdot \operatorname{Max} G(j) \quad(\lambda \geq 1),
$$

where $\operatorname{ComS}(j)$ is the characteristic value of row $j, \lambda$ is set as 1 in this paper. The maximum gray value $\operatorname{Max} V(j)$, minimum gray value $\operatorname{Min} V(j)$ and the maximum gradient value $\operatorname{Max} G(j)$ constitute a composited signal $\operatorname{ComS}(j)$, which is employed to indicate the distribution of structure planes along the depth direction in borehole camera image. During practical applications, in order to filter out the interference of individual spots, these described $\operatorname{Max} V(j), \operatorname{Min} V(j), \operatorname{Max} G(j)$ and $\operatorname{Com} S(j)$ signals are respectively independent filtering and denoising processing. Lastly, the composited signal $\operatorname{Com} S(j)$ can effectively and accurately highlight the regional distribution of structure planes.

\subsection{The regional division of structure planes}

The composited signal $\operatorname{ComS}(j)$ obtained above is an effective expression of the distribution region of structure planes in the image. To better processing and analysis, it is necessary to carry out threshold segmentation and binarization processing for the composited signal $\operatorname{ComS}(j)$. Since rock structure is complex and the rock mass may change, the background conditions of borehole camera image may also change along. Therefore, the threshold selection in the image needs to consider the local and whole condition. The global threshold of the composited signal is taken as the average of the whole region shown in Eq. (6). In addition, the average value of $w$ rows within current 
region denotes the local threshold shown in Eq. (7).

$$
\begin{gathered}
\overline{\operatorname{Com}_{\text {global }}}=\frac{1}{M} \sum_{j=0}^{M-1} \operatorname{ComS}(j) . \\
\overline{\operatorname{ComT}_{\text {local }}}=\frac{1}{2 w} \sum_{k=j-w}^{k+w} \operatorname{ComS}(k) \quad(w \in[10,100]) .
\end{gathered}
$$

The $M$ is the number of the whole composited signal. $\overline{\operatorname{ComT}_{\text {global }}}$ is the global threshold of $\operatorname{ComT}(j)$. The $w$ generally takes on 50 is an offset width along up and down of present line. $\overline{\operatorname{ComT}_{\text {local }}}$ is the local threshold of $\operatorname{ComT}(j)$. The sub-block signal $\operatorname{ComT}(j)$ is shown in Eq. (8), which is the binarization result of the composited signal $\operatorname{Com} S(j)$.

$$
\operatorname{Com} T(j)=\left\{\begin{array}{l}
1, \quad \operatorname{ComS}(j) \geq\left(\overline{\operatorname{Com}_{\text {global }}}+\overline{\operatorname{Com} T_{\text {local }}}\right) / 2 \\
0, \quad \operatorname{ComS}(j)<\left(\overline{\operatorname{Com} T_{\text {global }}}+\overline{\operatorname{Com} T_{\text {local }}}\right) / 2
\end{array} .\right.
$$

According to $\operatorname{Com} T(j)$, we can conveniently perform the positioning and analysis of the structure plane curves. When $\operatorname{ComT}(j)$ is not zero, it is recorded as the region of the structure plane. In practice, in order to avoid the loss of information that may exist, some possible small regions can be merged into a larger region. The division results of borehole camera image region are shown in Fig. 3 where the red region line is the start and end mark line of the block.

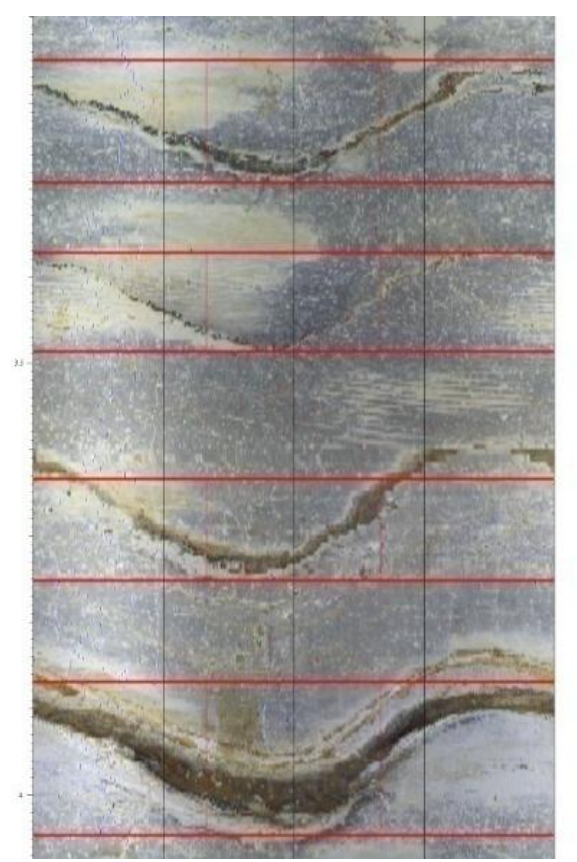

Fig. 3. Region division result of structure planes in a section borehole camera image. 


\subsection{Sine function iterative matching and interpretation}

From the above, the shape of structure planes in the image is similar to sine curve, so this paper adopts sine function as a template function. This step is to successively change the orientation $\alpha$ and the dip angle $\beta$ of the template function for each row in each region, and change step size can be set as $1^{\circ}$. Then, they are transformed into the corresponding phase $\theta$ and amplitude $A$ of sine curve. We adopt the maximum number of sine curve as the structure plane curve, that is, select the largest matched number in each row respectively. Finally, one or more optimal sine curves are screened out as the structure plane curve of this region.

According to the sub-block signal $\operatorname{Com} T(j)$, the size $W$ of the block width of the structure plane can be defined as the maximum dip angle $\beta$ of the structure plane, thus limiting the variation range of amplitude $A$ of sine curve. Again, based on the center position of structure plane, the valley values' position can be roughly determined. The approximate range of orientation $\alpha$ of the sine curve can be obtained, that is, the approximate range of phase angle $\theta$ of the template sine function is obtained. Lastly, the template sine function can be defined as Eq. (9), where $D$ is the borehole diameter, $W$ is the sub-block width, $\mathrm{k}$ is the resolution of the borehole camera system (DPBCS), $\alpha$ is the orientation and $\beta$ is the angle of structure plane.

$$
y(i)=j-\frac{D}{2 \mathrm{k}} \tan \beta \cdot \sin \left(i \cdot \frac{2 \pi}{N}+\theta\right), i \in[0, N-1], \theta \in(0,2 \pi), \beta \in\left[0, \frac{\mathrm{k} W}{D}\right) .
$$

In order to judge whether the point passed by or near the region, a threshold is employed to distinguish it when the template sine function is employed to match the structure plane. The threshold value is employed to express the characteristics of the region by using the average value of the pixel gray values in the region. The judging threshold of pixel value whether belongs to structure plane is defined as Eq. (10), where $m$ is the number of rows in the block, $n$ is the column number of the block, $i_{0}$ is the starting line of the block, $j_{0}$ is the starting column of the block. $T_{\text {gary }}$ is the judging threshold.

$$
T_{\text {gray }}(j)=\frac{1}{m n} \sum_{i=i_{0}}^{i_{0}+m} \sum_{j=j_{0}}^{j_{0}+n} f(i, j) .
$$

When template sine function $y(i)$ is employed to match of the pixel gray value, the total number of pixel points which satisfy the threshold condition is counted. The position, orientation and dip angle of the structure plane are also recorded. The total number of matched points in or near the structure plane are defined as a function 
$\operatorname{MaxN}(j)$, which records the maximum value of the total matched points of each row in the block.

It is obvious that sine curve with the maximum matching number can best reflect the features of the structure plane. The largest number of sine curve of the structure plane is the most consistent sine curve of the structure plane, which can be found basically in each row in the sub-block region. In a complex borehole camera image, uncertain factors such as rupture and perfunctory may generate multi-slit structure planes, so there may also be structure planes or fissures in the region where there are the largest points and the fastest changes. Therefore, according to the difference of the orientation or position of the sine curve, the maximum value of $\operatorname{MaxN}(j)$ is selected as the structure plane curve as shown in Fig. 4. It shows that $\operatorname{Max} N(j)$ exists 4 blocks and each block has one extreme point.

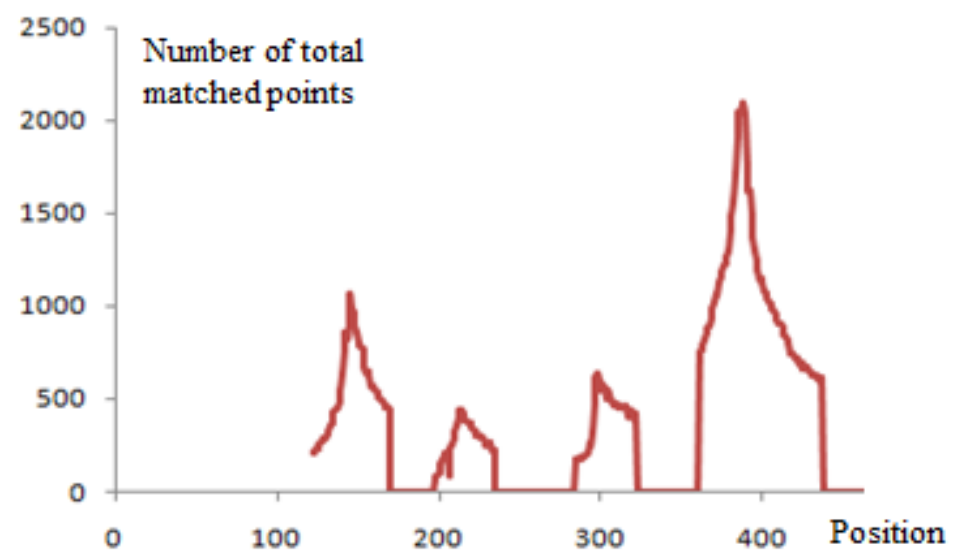

Fig. 4. The distribution of the total matched points from $\operatorname{MaxN}(i)$ signals.

The sine curve corresponding to the extreme points of Fig. 4 is selected as the structure plane curve of the region. The screening results of this region are shown in Fig. 5 and related parameters are shown in Table 1.

Table 1 Related parameters of structure plane curves

\begin{tabular}{ccccc}
\hline No. & Row & Orientation $^{\circ}$ & Dip angle $^{\circ}{ }^{\circ}$ & Matched points \\
\hline 1 & 145 & 108 & 6 & 1026 \\
2 & 213 & 111 & 6 & 437 \\
3 & 298 & 119 & 6 & 636 \\
4 & 387 & 118 & 8 & 2083 \\
\hline
\end{tabular}




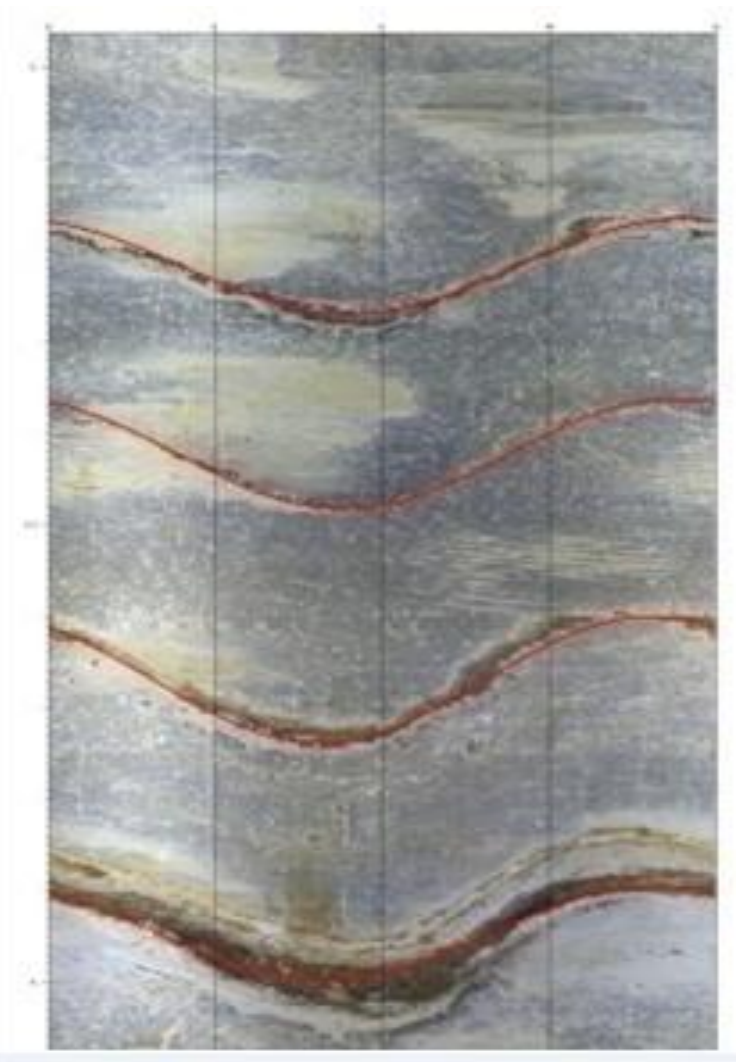

Fig. 5. The matched result between sine curves and structure planes in a selected part of borehole image.

\subsection{Parameter extraction and transformation}

In the above process, the finally obtained pixel coordinate is the row coordinate $j$ of sine curve at present analyzed region. The actual depth position Pos of the structure plane must be converted to the actual depth. Similarly, the finally obtained dip angle $\beta$ is exactly dip angle $\beta$ of the structure plane. The orientation $\alpha$ is the angle of the clockwise direction corresponded to by the sine curve trough and the north direction, which can be further converted into the actual engineering parameter.

The calculation method for the fracture width $d$ of structure plane is as follows: in each region, the sine curve of the structure plane is selected through the upper and lower moving,. When the sine curve and the upper boundary of the structure plane intersect, the minimal offset position of row coordinate is denoted as $j_{\min }$. When the sine curve and the lower boundary of the structure plane intersect, the maximal offset position of row coordinate is denoted as $j_{\max }$. The fracture width is the fluctuation range $\left(j_{\max }-j_{\max }\right)$ in the borehole image. Then the structural parameters such as position, 
orientation, dip angle and fracture width are obtained. And the data are saved in the database for an easier access. In the section of the borehole image Fig. 5, its structure plane parameters are shown in Table 2.

Table 2 Finally obtained parameters of structure planes through automatic interpretation

\begin{tabular}{ccccccc}
\hline No. & Depth $/ \mathrm{m}$ & Row & Matched points & Orientation $^{\circ}$ & Dip angle $^{\circ}$ & Fracture width $/ \mathrm{mm}$ \\
\hline 1 & -3.218 & 145 & 1064 & 161.3 & 47.8 & 9.2 \\
2 & -3.423 & 213 & 471 & 157.5 & 49.3 & 2.7 \\
3 & -3.678 & 298 & 673 & 150.2 & 51.7 & 12.5 \\
4 & -3.979 & 387 & 2194 & 152.6 & 56.4 & 23.8 \\
\hline
\end{tabular}

\section{Results and discussions}

\subsection{Automatic interpretation results}

Automatic interpretation method takes maximum gray value $\operatorname{Max} V(j)$, minimum gray value $\operatorname{Min} V(j)$, maximum gradient value $\operatorname{Max} G(j)$, composited signal $\operatorname{ComS}(j)$ and sub-block signal $\operatorname{Com} T(j)$ to effectively discover the structural plane in borehole camera image. These value functions effectively describe the distribution of structural planes. In order to solve the problem of multiple parallel or cross structure planes in a region, this paper selects the sine curve that has the maximum or extreme values of total matched number $\operatorname{Max} N(j)$ within the region as the structure plane curve. The difference of the position, phase angle and amplitude of the sine curve corresponds to the maximal or extreme of $\operatorname{MaxN}(\mathrm{j})$, which are employed to distinguish the multiple structure planes. When the maximum value of $\operatorname{Max} N(\mathrm{j})$, the phase and amplitude of the sine curve are close to each other, there are some parallel structure planes in the region. When the maximum value of $\operatorname{MaxN}(\mathrm{j})$, the position, phase and amplitude of the sine curve have the great differences, there are some cross structure planes in the region. The processing result of the cross structure planes in the same block is shown in Fig. 6a. The processing result of parallel structure planes in the same block is shown in Fig. 6b. 


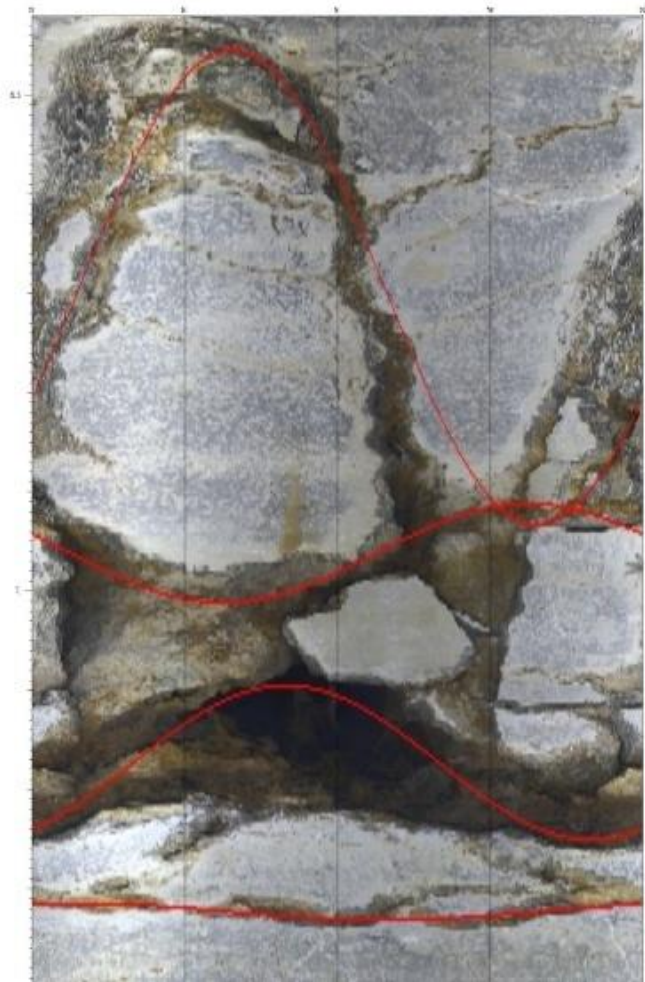

(a)

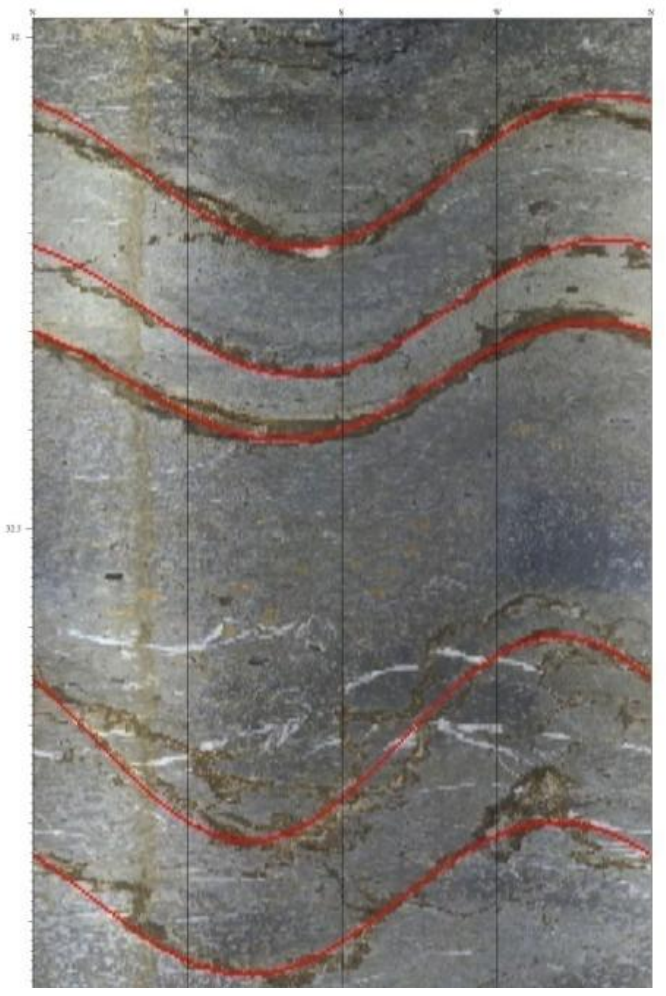

(b)

Fig. 6. Automatic interpretation results of multiply structural planes, (a) Crossed structural planes, (b) Paralleled structural planes.

The distribution of $\operatorname{MaxN}(j)$ in Fig.6a is shown as the curve of $\operatorname{MaxN}(j) \_$a in Fig. 7. The distribution of $\operatorname{Com} T(j)$ in Fig. 6a is shown as the curve ComT $(j) \_$a in Fig. 7. The curve of $\operatorname{Com} T(j) \_$a signal exists a block and 4 extreme points of curve $\operatorname{MaxN}(j) \_\mathrm{a}$ under the block $C o m T(j) \_$. The 4 extreme points of curve $\operatorname{MaxN}(j) \_$a correspond to 4 sine curves. The parameters of these 4 sine curves are used to represent the geometry parameters of structural planes in borehole camera image. They are shown as No. 1-4 in Table 3. The distribution of $\operatorname{MaxN}(j)$ in Fig. $6 \mathrm{~b}$ is shown as the curve of $\operatorname{MaxN}(j) \_b$ in Fig. 7. The distribution of $\operatorname{Com} T(j)$ in Fig. $6 \mathrm{~b}$ is shown as the curve $\operatorname{ComT}(j) \_\mathrm{b}$ in Fig. 7. The curve of ComT $(j) \_b$ exists two blocks. There are 3 extreme points of curve $\operatorname{MaxN}(j) \_\mathrm{b}$ under the first block of $\operatorname{ComT}(j) \_\mathrm{b}$ and 2 extreme points under the second block of $\operatorname{Com} T(j) \_$b. These 5 extreme points of curve $\operatorname{MaxN}(j) \_$b correspond to 5 sine curves. The relevant parameters of the 5 sine curves are also used to represent the geometry parameters of structural planes in borehole camera image. They are shown as No. 5-9 in Table 3. 


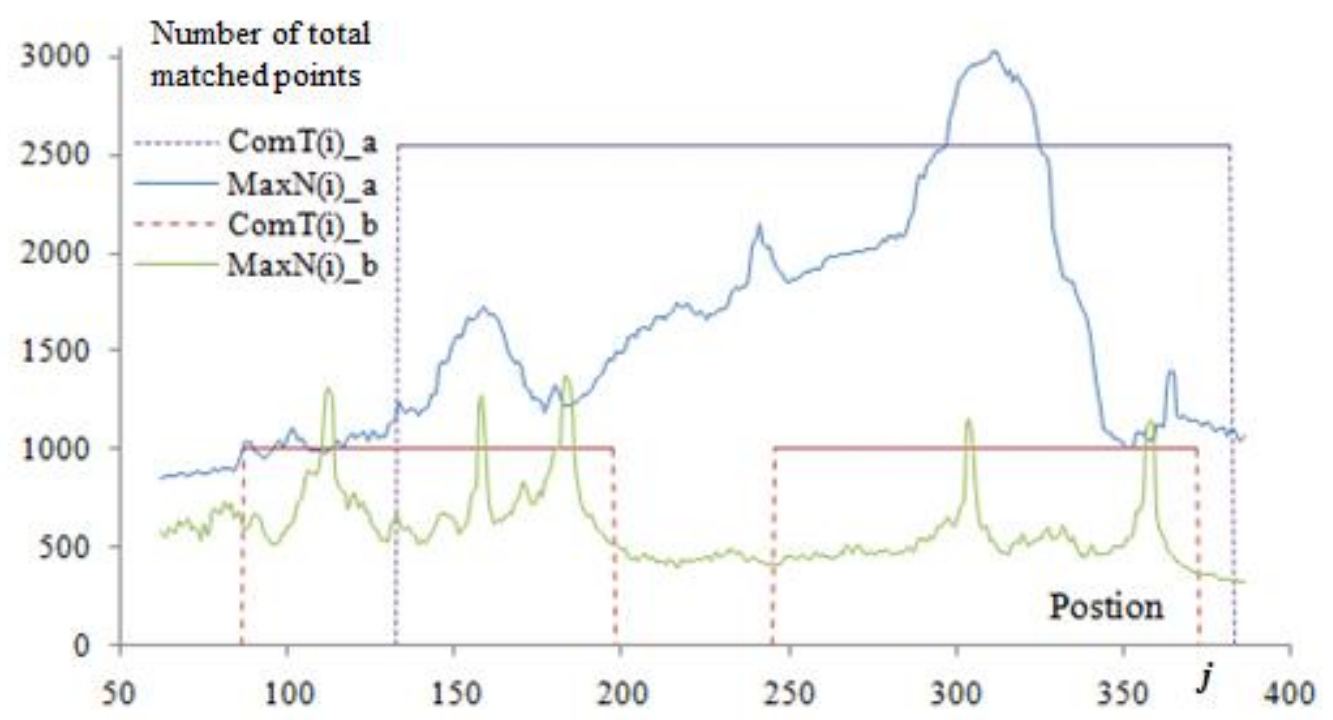

Fig. 7. Distributions of matched points signals $\operatorname{Max} N(j)$ and sub-block signals $\operatorname{Com} T(j)$ in the crossed and paralleled structural planes of Fig. 6 .

Table 3 Parameters of automatic interpretation for structure planes in Fig. 7

\begin{tabular}{cccccc}
\hline No. & Depth $/ \mathrm{m}$ & Matched point & ${\text { Orientation } /{ }^{\circ}}$ & Dip angle $/{ }^{\circ}$ & Fracture width $/ \mathrm{mm}$ \\
\hline 1 & -6.695 & 1721 & 332.4 & 79.2 & 22.6 \\
2 & -6.955 & 2143 & 157.3 & 47.5 & 20.3 \\
3 & -7.164 & 3022 & 300.6 & 59.6 & 98.5 \\
4 & -7.320 & 1392 & 62.2 & 10.3 & 9.2 \\
5 & -32.141 & 1315 & 115.7 & 58.4 & 13.4 \\
6 & -32.276 & 1270 & 116.8 & 57.2 & 8.3 \\
7 & -32.351 & 1370 & 124.1 & 55.8 & 24.8 \\
8 & -32.715 & 1151 & 114.6 & 65.6 & 7.6 \\
9 & -32.876 & 1149 & 145.3 & 60.7 & 8.5 \\
\hline
\end{tabular}

From the comparison of Fig. 5-7 and Table 2-3, one sub-block region of $\operatorname{MaxN}(j)$ can correspond to several extreme points, and every extreme point corresponds to a probable structure plane. The parameters from these probable structure planes are employed to select the best structure plane. As shown in Fig. 6, the red sine curve recognized automatically by this method is basically consistent with the original structure plane.

\subsection{Comparison with manual analysis}

From above, this method can automatically identify the structural features of the 
whole borehole camera image, and obtain some important parameters such as the central position, orientation, dip angle and fracture width of structural planes. In order to further illustrate the accuracy of automatic interpretation results, this paper employs a traditional method by manual analysis. Manual analysis method is as follows: with three control points given in the image by manual operation, use standard sine curve to fit the structural plane by computer, and then determine whether the fitting curve is consistent with the original structure plane or not. And then the central position, orientation, dip angle and fracture width calculate by manual operation. For the same section of borehole camera image, that is, Fig. 7a-b, the parameter extraction results by using manual analysis method are shown in Table 4.

Table 4 Related parameters of manual analysis for structure planes in Fig. 7

\begin{tabular}{ccccc}
\hline No. & Depth $/ \mathrm{m}$ & Orientation $^{\circ}$ & Dip angle $/{ }^{\circ}$ & Fracture width $/ \mathrm{mm}$ \\
\hline 1 & -6.664 & 331.7 & 79.4 & 21.4 \\
2 & -6.969 & 157.5 & 46.9 & 18.9 \\
3 & -7.171 & 301.1 & 58.7 & 99.3 \\
4 & -7.320 & 61.3 & 10.3 & 10.9 \\
5 & -32.183 & 116.5 & 57.6 & 14.1 \\
6 & -32.276 & 117.2 & 56.9 & 8.7 \\
7 & -32.344 & 124.3 & 55.8 & 24.5 \\
8 & -32.702 & 114.5 & 65.7 & 9.4 \\
9 & -32.881 & 144.8 & 61.1 & 9.8 \\
\hline
\end{tabular}

Due to the impact of human error and various uncertain factors, it is difficult to obtain an accurate interpretation result one time, so we have to repeat the manual analysis process and to obtain an average value. In Table 4, the result parameters from the manual analysis method are our averaged results. They are well consistent with the actual discontinuities in borehole image and they can be identified as standard parameters. Therefore, the comparison between the results of manual analysis method and the result of automatic interpretation method can reflect the interpretation rate and the accuracy of our method. The result of automatic interpretation method is shown in Table 3, which is compared with the data of Table 4. Their comparison results are shown in Table 5. 
Table 5 Comparison results of automatic interpretation and manual analysis

\begin{tabular}{ccccc}
\hline No. & Depth $/ \mathrm{m}$ & ${\text { Orientation } /{ }^{\circ}}$ & Dip angle $/{ }^{\circ}$ & Fracture width $/ \mathrm{mm}$ \\
\hline 1 & -0.031 & -0.7 & 0.2 & -1.2 \\
2 & 0.014 & 0.2 & -0.6 & -1.4 \\
3 & 0.007 & 0.5 & -0.9 & 0.8 \\
4 & 0.000 & -0.9 & 0.4 & 1.3 \\
5 & -0.042 & 0.8 & -0.8 & 1.5 \\
6 & -0.008 & 0.4 & -0.3 & 0.4 \\
7 & 0.007 & 0.2 & 0.0 & -0.3 \\
8 & -0.013 & -0.1 & 0.1 & 1.8 \\
9 & 0.005 & -0.5 & 0.4 & 1.3 \\
\hline
\end{tabular}

The results of Table 3 show that automatic interpretation method can identify all structure planes in borehole camera image. From Table 5, the automatic interpretation results are in good agreement with the actual structure planes and also the results of manual analysis method. From Table 5, the absolute deviation of depth is less than $0.05 \mathrm{~m}$, the absolute deviation of orientation is less than $1^{\circ}$, the absolute deviation of the dip angle is less than $1^{\circ}$ and the absolute deviation of fracture width is less than 2 $\mathrm{mm}$. Therefore, the automatic interpretation method of structural plane parameters in borehole camera images can meet the actual engineering requirements.

\subsection{Discussion about the limitation}

Due to the diversity of rock structures, a large amount of noise signals are also present. With appropriate signal filtering and smoothing, the main features of the structural planes can be also captured by automatic interpretation method. However, some discontinuous or broken structural planes can also be found in actual borehole image, which may lead to partial loss of structural planes. When the borehole camera image is segmented by vertical projection, there partly are the cross structure planes or the relatively parallel connection of structure planes in the borehole camera image. When successively matching structure of feature points using template sine function, each row of each block selects a sine curve with the most matched points. These sine curves are the maximum or extreme values of total matched number $\operatorname{MaxN}(j)$, and they still need to be further screened. However, there are many closely-linked parallel structure planes or broken planes, and some structure planes cross each other, which cannot be separated from each other. A sine curve that has the largest matched points' number may still cause the loss of probable structure planes in the region as shown in 
Fig. 8, which are the automatic interpretation for structure planes in cross-cutting and overlaying borehole images. From Fig. 8, most of main structural planes are detected and marked in red as standard sine curve. It has met the basic requirements of actual borehole image analysis from engineering application. However, we can find there are some fractures which can't be detected. These fractures are appeared to be cemented by calcite or are some isolated faults, weak surfaces, weak joints and weak zones. For these weak, isolated or small structural planes which are nonstandard sine curve band, this automatic method still need to be improved.

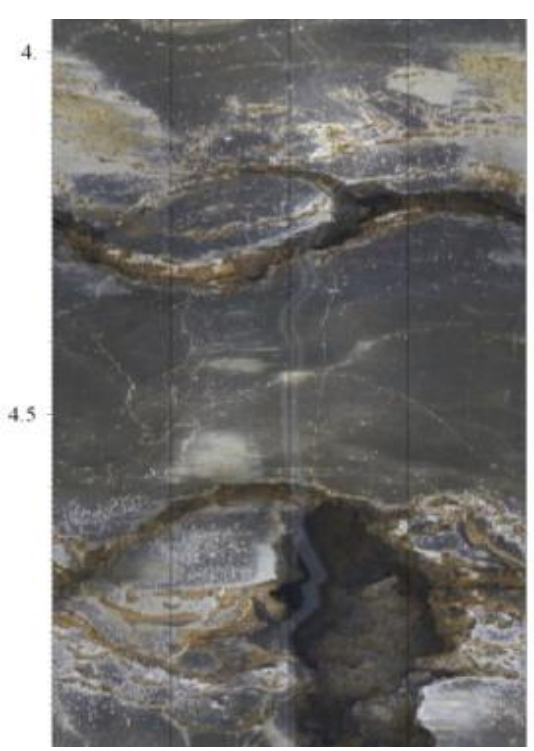

(a)

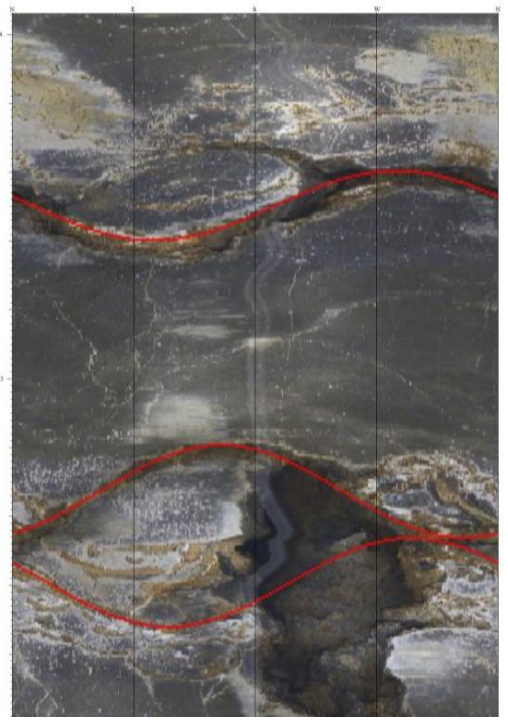

(c)

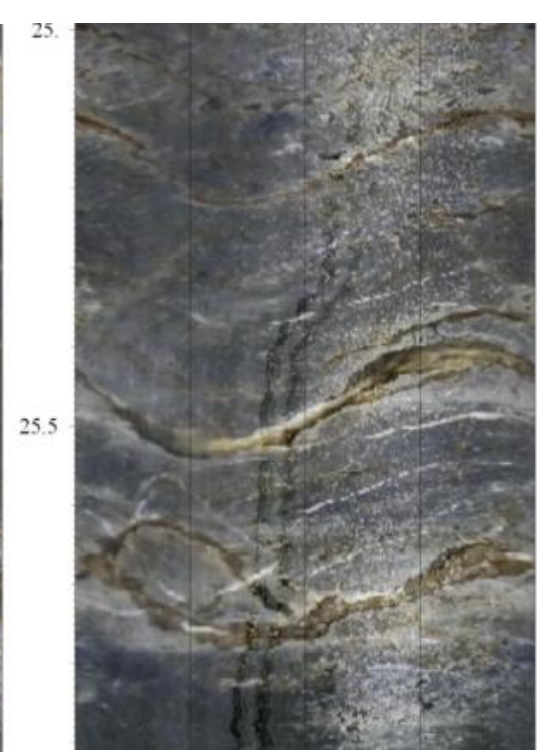

(b)

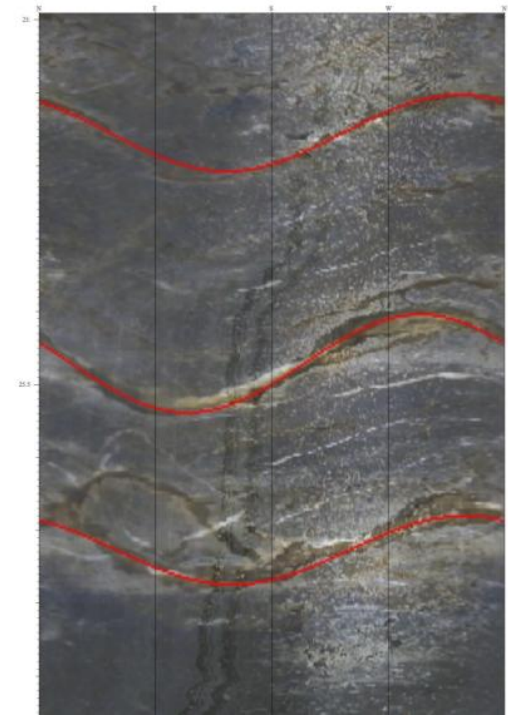

(d)

Fig. 8. The automatic interpretation results of cross-cutting and overlaying borehole images, (a) The original cross-cutting structural planes, (b) The original overlaying structural planes by dirty zone, (c)

The automatic interpretation results of (a), (d) The automatic interpretation results of (b). 
This automatic interpretation method can automatically detect the whole borehole camera image and accurately extract the structural plane parameters without manual analysis or semi-automatic operation. However, for some complex imaging logging data and acoustic or resistivity image log, or the borehole image logs with continuous panels, or the borehole image that is worked for different setting features at different locations, the automatic interpretation of these borehole images may be failed at present. Therefore, we have taken some effective pre-treatment methods to translate these complex cases into multiple simple cases, which are easy to be deal with in a particular region. Lastly, these images can be automatically recognized respectively. In actual application, the pre-treatment methods are complex and need to meet kinds of cases, which is a tough work. Some opened or cemented fracture, joint and bedding planes may can't detect by this method because of narrow area or weak pixel values or small number imaging points in the image when compared with the main fractures, even though they can observed on image logs. This automatic interpretation method is for the detection of main fractures or structural planes in the image, but I think we can provide a better solution for complex cases and engineering application in future.

Another, because we can't obtain other borehole imaging logs such as gamma log, density log and resistivity log data, this method is just tested well in the optical image, for example, the borehole camera image obtained by our Digital Panoramic Borehole Camera System (DPBCS). Those resistive or conductive borehole imaging logs are important part of borehole imaging logging data. We will test them if possible in future. At present, the interpretation of all simple and obvious structural planes in the entire borehole camera images can be automatically finished within an acceptable time without the operations of people. It has been used in actual borehole images for saving time in drilling engineering.

\section{Conclusions}

In this paper, a method of automatic continuous interpretation is proposed for the parameter extraction of structure plane in the whole borehole camera image. In this method, the maximum, minimum gray values and the maximum gray gradient value of each row of the borehole camera image are extracted and analyzed, and a new feature signal is introduced. In addition, the proposed method directly employs the template sine function to iterate through all the possible structures in the matching region, and then filter out the best matching parameters, which is employed as the 
characteristic curve of the structure plane. This method is simple and reliable. By optimizing the region partition and matching result selection method, the method can perform the automatic interpretation of structure plane and intelligent extraction of geometric parameters in borehole camera image. The automatic interpretation results are in good agreement with the structure planes in the borehole camera image. This method can perform the intelligent analysis and automatic interpretation of structural planes for borehole camera image with stable and reliable interpreted results, and the efficiency of drilling camera image analysis is high. It provides a simple and reliable method for the processing and application of borehole camera image data from actual drilling engineering.

\section{Acknowledgments}

This paper was supported by the National Science Foundation of China (Grant No. 41402278 and No. 41372317$)$.

\section{References}

Assous, S., Elkington, P., Clark, S. and Whetton, J., 2014. Automated detection of planar geologic features in borehole images. Geophysics, 79(1): D11-D19.

Bae, D., Kim, K., Koh, Y. and Kim, J., 2011. Characterization of joint roughness in granite by applying the scan circle technique to images from a borehole televiewer. Rock Mechanics and Rock Engineering, 44(4): 497-504.

Cunningham, K.J., Carlson, J.I. and Hurley, N.F., 2004. New method for quantification of vuggy porosity from digital optical borehole images as applied to the karstic Pleistocene limestone of the Biscayne aquifer, southeastern Florida. Journal of Applied Geophysics, 55(1-2): 77 - 90.

Djurhuus, J. and Aadn O Y, B.S., 2003. In situ stress state from inversion of fracturing data from oil wells and borehole image logs. Journal of Petroleum Science and Engineering, 38(3): 121 --130.

Genter, A. et al., 1997. Comparative analysis of direct (core) and indirect (borehole imaging tools) collection of fracture data in the Hot Dry Rock Soultz reservoir (France). Journal of Geophysical Research: Solid Earth, 102(B7): 15419--15431.

Glossop, K., Lisboa, P.J.G., Russell, P.C., Siddans, A. and Jones, G.R., 1999. An Implementation of the hough transformation for the identification and labelling of fixed period sinusoidal curves. Computer Vision and Image Understanding, 74(1): 96 - 100.

Han, Z., Wang, C., Liu, S. and Zhu, H., 2013. Research on connectivity of deep ore-lodes of borehole based on digital borehole camera. Disaster Advances, 6(8): 41-46. 
Hurich, C. and Deemer, S., 2013. Combined surface and borehole seismic imaging in a hard rock terrain: A field test of seismic interferometry. Geophysics, 78(3): B103-B110.

Lofi, J. et al., 2012. Geological discontinuities, main flow path and chemical alteration in a marly hill prone to slope instability: Assessment from petrophysical measurements and borehole image analysis. Hydrological Processes, 26(14): 2071--2084.

Prensky, S.E., 1999. Advances in borehole imaging technology and applications. Geological Society, London, Special Publications, 159(1): 1-43.

Schepers, R., Rafat, G., Gelbke, C. and Lehmann, B., 2001. Application of borehole logging, core imaging and tomography to geotechnical exploration. International Journal of Rock Mechanics and Mining Sciences, 38(6): 867 - 876.

Thapa, B.B., Hughett, P. and Karasaki, K., 1997. Semi-automatic analysis of rock fracture orientations from borehole wall images. Geophysics, 62(1): 129-137.

Wang, C., Ge, X. and Bai, S., 2002. Study of the digital panoramic borehole camera system. Chinese Journal of Rock Mechanics and Engineering, 21(3): 398-403.

Wang, C., Hu, P. and Sun, W., 2010. Method for evaluating rock mass integrity based on borehole camera technology. Rock and Soil Mechanics, 31(4): 1326-1330.

Williams, J.H. and Johnson, C.D., 2004. Acoustic and optical borehole-wall imaging for fractured-rock aquifer studies. Journal of Applied Geophysics, 55(1-2): 151 - 159.

Zohreh, M., Junin, R. and Jeffreys, P., 2014. Evaluate the borehole condition to reduce drilling risk and avoid potential well bore damages by using image logs. Journal of Petroleum Science and Engineering, 122: $318-330$. 